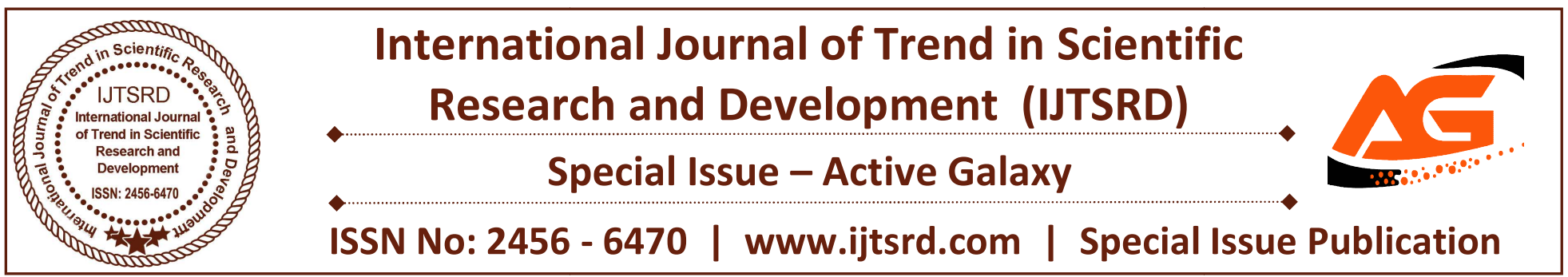

\title{
Wearable Artificial Kidney: A Dialysis Machine to Clean Blood
}

\author{
Saranya. C. $\mathbf{S}^{1}$, Dr. Kala. $\mathbf{S}^{2}$ \\ ${ }^{1} \mathrm{ME}$ - Applied Electronics, ${ }^{2}$ Professor and Head \\ Sri Subramanya College of Engineering and Technology, \\ Palani, Dindigul, Tamil Nadu, India
}

\begin{abstract}
Elderly individuals with constant assault are winding up increasingly various in our nation. In Mexico, an interminable assault that is among the best ten reasons for mortality. Individuals who endure Chronic Kidney Disease (CKD) must be on a consistent audit to keep up illness control and observing. In hemodialysis, a manufactured kidney (hemodialyzer) is utilized to expel waste and additional chemicals and fluid from your blood. To get your blood into the fake kidney, the specialist needs to influence an entrance (to entrance) into your veins. This is finished by a minor task to your arm or leg.
\end{abstract}

Some of the time, an entrance is made by joining a conduit to a vein under your skin to make a greater vein called a fistula. In any case, if your veins are not worthy of a fistula, the specialist may utilize a delicate plastic tube to join a supply route and a vein under your skin. This is known as a unite. Sometimes, an entrance is made by methods for a thin plastic tube, called a catheter, which is embedded into a huge vein in your neck. This sort of access might be transitory, yet is infrequently utilized for long haul treatment.

Keywords: Kidney Disease, hemodialyzer, Peritoneal, kidney

\section{INTRODUCTION}

In recent years, kidney renal failure is found to be a major disease in the developed and developing countries. Various therapy methods adopted for renal failure are Haemodialysis, Peritoneal dialysis, and kidney transplantation [1]. In the above three methods Hemodialysis therapy method is accepted to be the best treatment method by most countries for renal failure. Hemodialysis working [2]

A Hemodialysis machine has a dialyzer filter which cleans the blood.

$>$ The doctor makes an access into our blood vessels. This is done with minor surgery on the arterio-venous fistula, usually to our arm. Then blood is taken into the dialyzer.

$>$ The dialyzer has two parts one part for our blood and the other part for a fluid called dialysate. These two parts are separated by a thin semi-permeable membrane.

$>$ Smaller waste products such as urea and extra fluid pass through the membrane and are removed.

$>$ Blood cells, protein and other important things remain in our blood because they are too big to pass through it.

Hemodialysis is the method to access the blood via two thin needles or a central venous catheter, and then it is connected to the dialysis lines. High blood flows circulate the blood to the dialyser, for cleansing. Haemodialysis carries a risk of blood spillage, whether it is done at home or in the hospital [3]. This may occur because of:

Accidental dislodgement of the needles (the venous needle creates the greatest blood loss)

$>$ Disconnection of the needles or catheter from the dialysis lines

$>$ Disconnection of the lines from the dialyser. An unfortunate blood leakage during 
hemodialysis therapy is at least inconvenient and messy, but at worst it could be fatal.

Even though Hemodialysis is the best treatment method for Kidney Renal failure the various technical problems associated with Hemodialysis are Venous Needle Dislodgement (VND), blood clotting, power failure, air in blood lines, dialyzer reaction. Out of the above listed technical problems in Hemodialysis, VND is found to be a potentially serious complication and as a result of VND there will be the blood leak occurs during Hemodialysis process. Thus blood leakage due to VND in hemodialysis is the most complicated problem and should be avoided during the Hemodialysis process [4]. So it is considered to be important to monitor the blood leaking condition during the hemodialysis treatment. In this study, an Infra-red sensor is used for detecting the blood leakage and combined with a Bluetooth module for the function of wireless transmission. IR sensor has the capability to measure a short distance with accuracy up to $0.5 \mathrm{~mm} 2$ [5], which is better compared to other sensors. IR sensor gives simple installation, small in size, lightweight, portable, and only requires a few components for its operation [6]. The sensor used here is to detect the occurrence of blood due to the sensitivity to the object reflectance properties. An absorbent material placed at the sensing region can be used for adsorbing the leaking blood. When the red coloured liquid (red ink representing actual blood) drops at the edge of absorbent material, the absorbent material adsorbs the red ink due to the capillarity action. At this point the red ink quickly accumulates the entire surface of the absorbent material which makes a change in light penetration when light falls on it. The sensing part of the sensor will sense the light changes and the original voltage signal of the sensor alters accordingly [7].

\section{RELATED WORK}

In end-stage kidney disease, the kidneys no longer adequately clear the blood of wastes and excess fluids. Most individuals with end-stage kidney disease receive dialysis treatment to compensate for some of the lost kidney function by removing excess fluids and metabolic wastes - such as urea - that accumulate in the blood. Although dialysis removes fluids and wastes, it does not replace the kidneys' role in regulating metabolism, endocrine function, and homeostasis (maintaining stable physiological processes such as blood pressure, electrolyte balance, acid-base balance, and temperature).

Providing patients who have end-stage kidney disease with more frequent and longer dialysis sessions can improve blood pressure control; weight and nutritional status; reduce stress on the heart; and clear the blood of toxins, wastes, and excess fluids at a more gradual rate that more closely resembles the continuous process performed by healthy kidneys.

Unfortunately, because dialysis is an expensive treatment and dialysis centre capacity is limited, health care systems often lack the resources to provide more frequent or longer treatment sessions to patients. Moreover, the changes patients must make to their diets, and the need to travel to receive dialysis treatment, negatively affect the quality of patients' daily lives.

Research is underway to develop a wearable artificial kidney that would allow individuals to receive dialysis as they go about their daily activities, improving the removal of wastes and fluids through more frequent, longer, or continuous dialysis sessions. This could improve patient health while reducing the impact of treatment on quality of life.

Dialysis is a life-sustaining treatment for patients with end-stage kidney disease. In a different context, for many patients this treatment is the focal point around which their life revolves, not only due to the time spent travelling to and from treatment sessions and the time dedicated to the dialysis treatment itself, but also due to the accompanying dietary and fluid restrictions and medication burden.

Wearable and portable dialysis devices could potentially improve patient quality of life by allowing patients to continue with their daily activities of life while undergoing dialysis, as well as by looseningor removing entirely-dietary and fluid restrictions and reducing pill burden.

Advances in nanotechnology manufacturing coupled with advances in electronics and miniaturisation have allowed a new generation of wearable and portable dialysis devices to be developed which are now undergoing large animal and patient clinical trials.

We are therefore potentially at a new dawn in the treatment of dialysis patients with the first 
generation of wearable and portable dialysis devices, which may well revolutionise the treatment and quality of life for patients with end-stage kidney disease.

\section{PROPOSED SYSTEM}

Patient having the debased blood and it can be gathered in Blood Purifier tank. After gathering the sullied blood it has some ailment like creatinine and urea, and so forth. For evacuating those infections we need to warm by utilizing 2 cathodes are embedded in the purifier tank and temperature can be observed by utilizing LM35 as Temperature sensor. After completely purged it can send to the patient through pumping motor. while sending the blood can check whether it can be spilled or not by utilizing Photo interrupter Tx and Rx.

Photo interrupter is utilized as a sensor for identifying the blood spillage and joined with a Bluetooth for the capacity of remote transmission. Besides, through our circuit plan, the blood spillage indicator can be coordinated on an armed jewelry, which is a basic and reasonable approach to screen the spillage of blood amid the hemodialysis treatment. If the locator detects a spillage of blood, the alarm messages will be sent through Bluetooth.

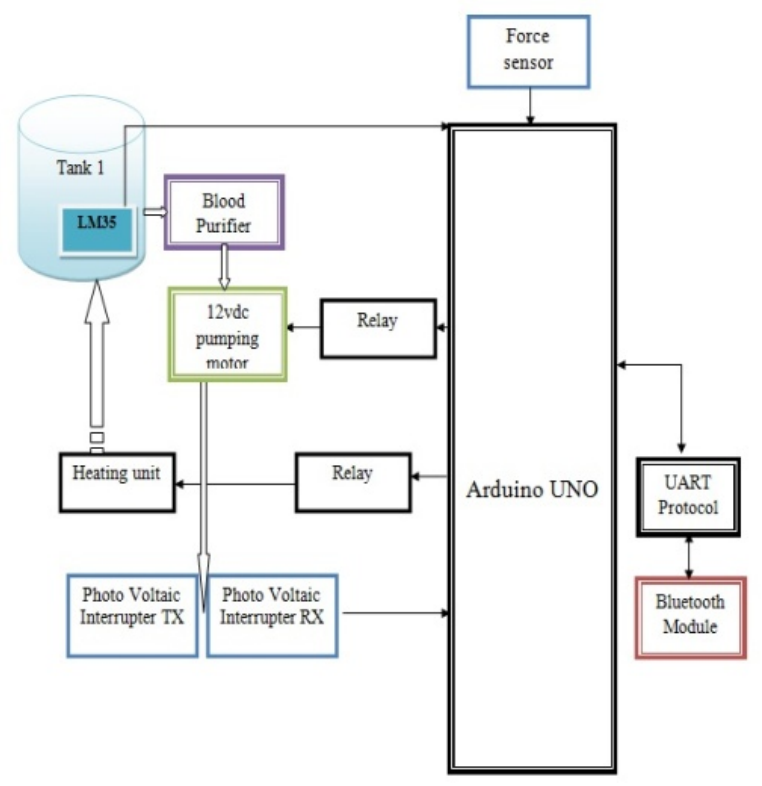

Fig1 : proposed system

\section{CONCLUSION}

A basic estimating framework for early discovery of hemolysis in HD is proposed. All in all, much work and numerous tests will be expected to examine the impacts of varieties in channel, patient, and treatment settings on the $\mathrm{R}$ work before the proposed estimating framework can be prepared for routine clinical use in HD, however these first outcomes recommend that hemolysis can be distinguished from the optical examination of the DF, in this way permitting decrease in the serious wellbeing hazard for patients related with hemolysis in HD medications.

\section{REFERENCES}

1) S. Cattini and L. Rovati, "Design and performance of an $\mathrm{Hb}$ measuring system: Viability of its use in a haemodialysis," in Proc. IEEE Int. Instrum. Meas. Technol. Conf. (I2MTC), May 2015, pp. 1147-1152.

2) S. Cattini, L. Rovati, M. Bernabei, D. Cianciavicchia, P. Monari, and A. Sicuri, "A simple measuring system for early detection of haemolysis during haemodialysis," in Proc. IEEE Int. Instrum. Meas. Technol. Conf. (I2MTC), May 2014, pp. 282-287.

3) B. H. Brown and R. H. Smallwood, Medical Physics and Physiological Measurement. Oxford, U.K.: Blackwell, 1981.

4) H.-D. Polaschegg, "Red blood cell damage from extracorporeal circulation in hemodialysis," Seminars Dialysis, vol. 22, no. 5, pp. 524-531, 2009.

5) S. J. Sweet, S. McCarthy, R. Steingart, and T. Callahan, "Hemolytic reactions mechanically induced by kinked hemodialysis lines," Amer. J. Kidney Diseases, vol. 27, no. 2, pp. 262-266, 1996.

6) R. Duffy et al., "Multistate outbreak of hemolysis in hemodialysis patients traced to faulty blood tubing sets," Kidney Int., vol. 57, no. 4, pp. 16681674, 2000.

7) Biological Evaluation of Medical Devices-Part 4: Selection of Tests for Interactions With Blood, ISO Standard 10993-4:2002, International Organization for Standardization, Geneva, Switzerland, 2002.

8) R. A. Sherman, J. T. Daugirdas, and T. S. Ing, "Complications during hemodialysis," in Handbook of Dialysis, 4th ed. Philadelphia, PA, USA: Lippincott Williams \& Wilkins, 2006, ch. 10. 


\section{Special Issue - Active Galaxy}

9) C. A. Schaer et al., "Mechanisms of haptoglobin protection against hemoglobin peroxidation triggered endothelial damage," Cell Death Differentiation, vol. 20, pp. 1569-1579, Aug. 2013.

10) G. Murcutt et al., "Guarding against hidden haemolysis during dialysis: An overview," J. Renal Care, vol. 33, no. 4, pp. 191-195, 2007.

11) V. F. Fairbanks, S. C. Ziesmer, and P. C. O'Brien, "Methods for measuring plasma hemoglobin in micromolar concentration compared," Clin. Chem., vol. 38, no. 1, pp. 132-140, 1992.

12)R. A. Malinauskas, "Plasma hemoglobin measurement techniques for the in vitro evaluation of blood damage caused by medical devices," Artif. Organs, vol. 21, no. 12, pp. 1255-1267, 1997.

13) G. B. van den Berg and C. A. Smolders, "Flux decline in ultrafiltration processes," Desalination, vol. 77, pp. 101-133, Mar. 1990.

14) H. P. Erickson, "Size and shape of protein molecules at the nanometer level determined by sedimentation, gel filtration, and electron microscopy," Biological Procedures Online, vol. 11, no. 1, pp. 32-51, 2009.
15) A. Barwicz, M. B. Slima, J. Barwicz, and P. Tancrede, "Signal processing support for the evaluation of blood toxic oxidation activity," IEEE Trans. Instrum. Meas., vol. 49, no. 3, pp. 541-545, Jun. 2000.

16) M. Zhang, D. N. Wang, W. Jin, and Y. Liao, "Wavelength modulation technique for intracavity absorption gas sensor," IEEE Trans. Instrum. Meas., vol. 53, no. 1, pp. 136-139, Feb. 2004.

17) G. Salvatori, K. L. Suh, R. R. Ansari, and L. Rovati, "Instrumentation and calibration protocol for a continuous wave near infrared hemoximeter," IEEE Trans. Instrum. Meas., vol. 55, no. 4, pp. 1368-1376, Aug. 2006.

18) R. Z. Morawski and A. Miekina, "Improving absorbance spectrum reconstruction via spectral data decomposition and pseudo-baseline optimization," IEEE Trans. Instrum. Meas., vol. 58, no. 3, pp. 691-697, Mar. 2009.

19) L. Rovati and S. Cattini, "UV-LEDs for monitoring dialysis adequacy," IEEE Trans. Instrum. Meas., vol. 58, no. 5, pp. 1720-1726, May 2009. 